\title{
Gasping for Air, a Case Study of an Obstructing Thyroid Gland Mass
}

\author{
Aakash Trivedi OMS-IV*, Aline Dang, DO, Clive Persaud, DO and Derick Christian, MD
}

St. Joseph's University Medical Center, USA

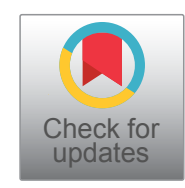

\begin{abstract}
Hürthle cell metaplasia of the thyroid gland can present with a variety of benign or malignant characteristics. At times, it can progress to a large mass causing a myriad of symptoms including dysphagia, vascular compression, and respiratory distress. We present a case of a 73-year-old woman with untreated, longstanding Hürthle cell metaplasia and fibrosis leading to significant airway obstruction. Because of these conditions, a thyroidectomy in a drastically distorted anatomical field was performed. Over time, different surgical approaches as well as nonsurgical management have been developed for thyroid disease. This case reinforces the need for surgical thyroidectomy due to the progressive nature of thyroid disease and simultaneous airway compromise.
\end{abstract}

\section{Background}

The term "Hürthle cell" has been used to describe follicular-derived epithelial cells with oncocytic cytology, otherwise known as an epithelial cell with acidophilic cytoplasm. This was first coined by Max Askanazy in 1898 in patients with Graves' disease. The characteristic features of Hürthle cells include distinct cell borders, abundant eosinophilic cytoplasm, numerous mitochondria, and a prominent nucleolus all of which contribute to the cell's size and staining characteristics [1]. We report a case of resection of Hürthle cell metaplasia and fibrosis of the left thyroid gland in a patient for over 25 years with findings of grossly distorted anatomy and complex surgical dissection.

\section{Case Presentation}

A 73-year-old Caucasian female with a past medical history of untreated neck mass and long-term tobacco use presented to the emergency department at St. Joseph's University Medical Center after being found unresponsive by family. The patient was in respiratory distress with findings of a large mass on the anterolateral neck. The patient was subsequently intubated and evaluated. CT scan of the neck was performed with findings of massive enlargement of the entire thyroid, diffuse nodular heterogeneity, significant narrowing of the airways at the level of the oropharynx and hypopharynx. The left lobe of the thyroid measured $6.7 \times 10.2 \times 11 \mathrm{~cm}$, the isthmus was $6.4 \mathrm{~cm}$, and the right lobe was $4.6 \times 3.6 \times 12.4 \mathrm{~cm}$ (Figure 1 and Figure 2). There was extension of the thyroid within the left paratracheal space into the superior mediastinum and trachea displacement to the right.

Biopsy of the mass was performed with findings significant for benign thyroid tissue with prominent Hürthle cell metaplasia and fibrosis. Findings were suggestive of sclerosing Hashimoto's Thyroiditis. Surgical resection was recommended due to airway obstruction.

\section{Surgical Procedure}

Surgical resection was initiated with an elongated cervical incision to accommodate the previously identified bilobed prominent left thyroid mass. Dissection was carried down through the attenuated platysma, at which point the left thyroid mass was encountered. The strap muscles were displaced laterally. The mass was carefully mobilized laterally and posteriorly with findings of gross displacement of the esophagus and trachea to the posterior right and obliteration of the expected left tracheoesophageal groove, subsequently requiring division of the left recurrent laryngeal nerve due to tumor invasion (Figure 3). Complete mobilization of what was identified as the left superior thyroid lobe was obtained and the mass was transected at the isthmus leaving a small amount of thyroid tissue inferiorly extending into the mediastinum. This mass was $17 \times 9 \mathrm{~cm}$ (Figure 4).

The second lobe of the mass was slightly inferior and right of the previous mass. It was determined that this was the inferior lobe of the left thyroid which had been displaced by

*Corresponding author: Aakash Trivedi OMS-IV, St. Joseph's University Medical Center, USA

Accepted: October 15, 2020

Published online: October 17, 2020

Citation: Trivedi A, Dang A, Persaud C, et al. (2020) Gasping for Air, a Case Study of an Obstructing Thyroid Gland Mass. J Surgical Endocrinol 2(1):46-48 


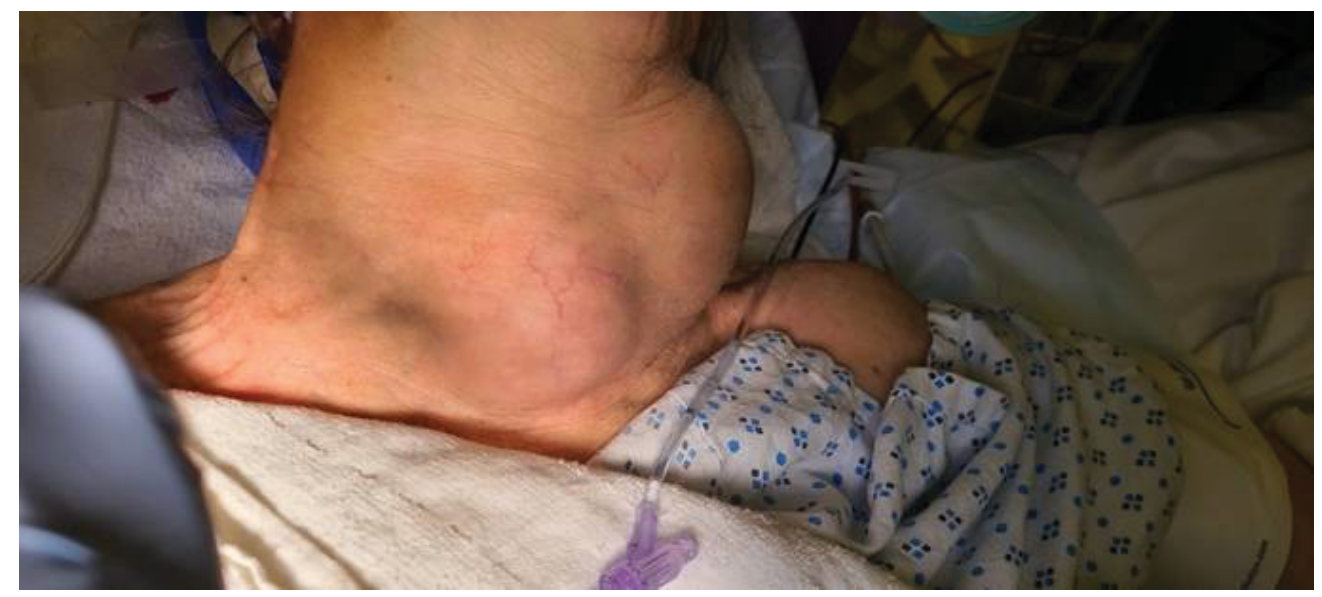

Figure 1: Gross anterior view of thyroid mass.

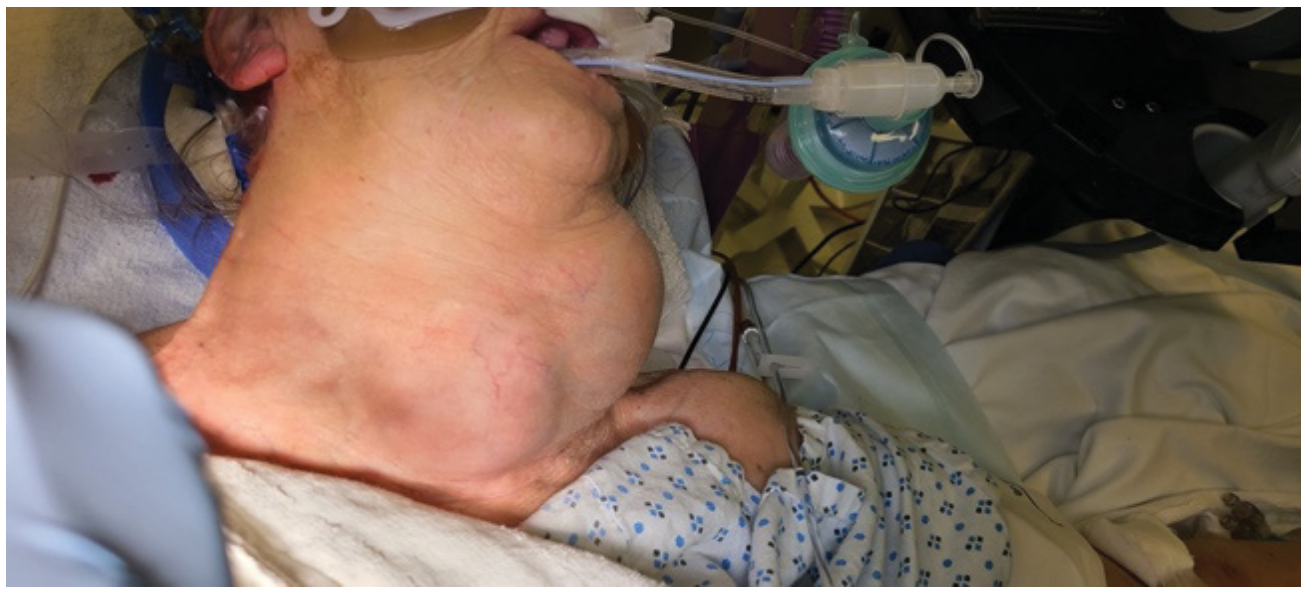

Figure 2: Gross anterolateral view of thyroid mass.

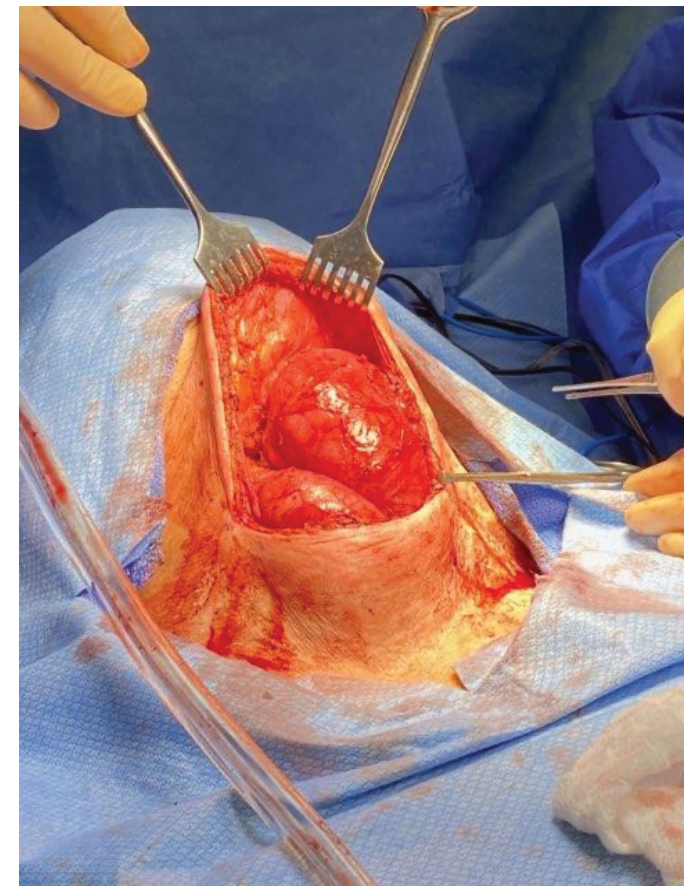

Figure 3: Intraoperative bilobed mass.

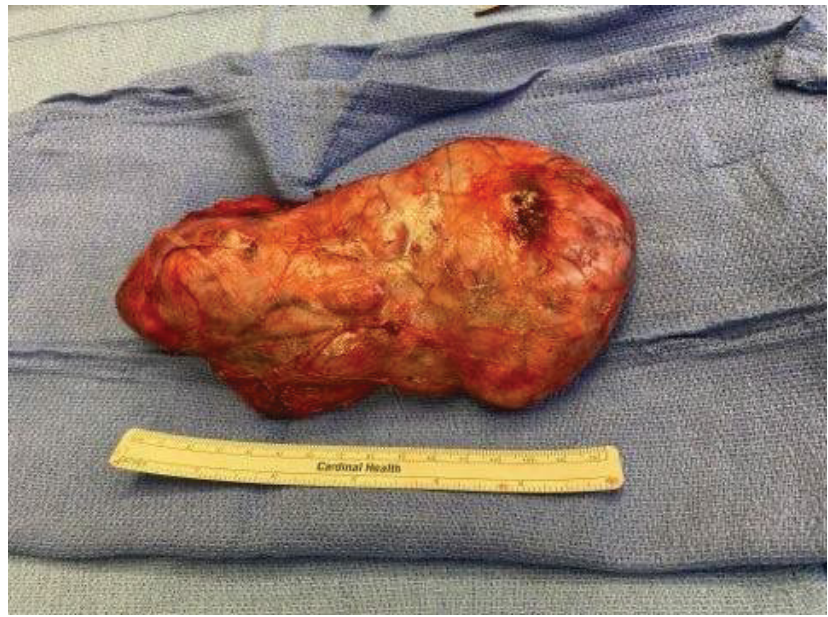

Figure 4: Resected left superior thyroid lobe and isthmus.

the superior lobe of the left thyroid. The inferior lobe was then mobilized and partially resected (Figure 5). This mass was $5 \times 7 \mathrm{~cm}$. Final pathology revealed a sclerosing variant of Hashimoto's Thyroiditis in both resected masses. The submitted surgical sections showed thyroid follicles separated by abundant dense collagenous fibrosis. Prominent lympho- 


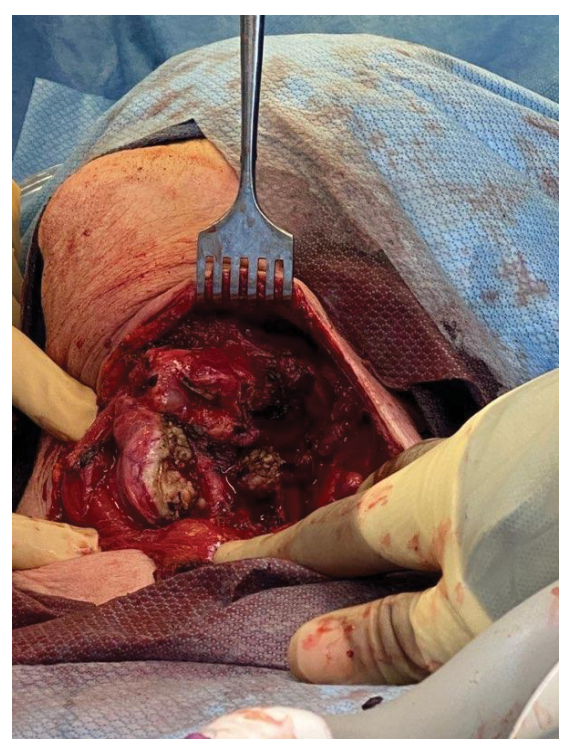

Figure 5: Anterior view of cervical surgical resection site.

plasmacytic infiltrate surrounded and infiltrated the lobules. Follicular cells showed extensive Hürthle cell metaplasia and atrophic changes.

Due to the complicated and distorted anatomy and previous transection of the left recurrent laryngeal nerve, the right lobe of the thyroid was left intact without exploration. The wound was closed without complication.

\section{Discussion}

In the thyroid gland, the two main tumors associated with Hürthle cells are Hürthle cell adenomas and Hürthle cell carcinomas. However, oncocytic metaplasia can be appreciated on a spectrum, ranging from benign adenomatous nodules and Hashimoto thyroiditis to papillary thyroid carcinoma [2]. Thyroid surgery has advanced tremendously over the last 30 years. Currently, there are minimally invasive and extracervical approaches that create technical ease, improve patient safety and satisfaction, and provide cost-effectiveness. Some of these approaches include trans-axillary, breast, postauricular, and transoral routes [3].

With proper preoperative planning, nonurgent timing, and relatively predictable anatomy, minimally invasive or extra-cervical approaches can be used for a thyroidectomy. This may have allowed for improved postoperative outcomes as well as identification of sensitive structures. However, in our case, the patient presented with over 25 years of untreated thyroid metaplasia and fibrosis. This led to not only a vastly distorted anatomical field, but also the need for urgent resection due to airway obstruction.

Moreover, recent literature has described using a median sternotomy as an approach for a surgical resection of goiter. The indications for this type of procedure include extension of the goiter below the aortic arch, large thyroid tissue extending towards the tracheal bifurcation, and ectopic thyroid tissue in the mediastinum. These substernal goiters can cause respiratory distress, dysphagia, vascular compression, and potential sudden death. A median sternotomy can be used alongside a cervical incision to remove certain types of thyroid masses [4].

In this case, the patient was prepared for possible median sternotomy. However, due to medical comorbidities and ability to mobilize a sufficient inferior portion of the superior thyroid mass, this was circumvented. Due to the need to divide the left recurrent laryngeal nerve and gross distortion of normal anatomy, it was determined that there was no benefit to exploration of the right thyroid gland after resection of the left inferior thyroid mass to avert the possibility of hoarseness, aphonia, vocal cord paralysis, and respiratory distress. For a healthier, more suitable patient with an untreated neck mass for greater than 25 years, a substernal approach might be used in order to achieve total resection of the left sided neck mass.

In stable patients without airway obstruction due to a thyroid mass or after incomplete resection, one might consider nonsurgical treatment options for Hürthle cell metaplasia. One treatment option is external radiotherapy for palliative relief from symptomatic metastases, control recurrent tumors, and prevent recurrence of advanced resected tumors. Another option is chemotherapy including multikinase inhibitors, selective kinase inhibitors, and combination therapies [5]. Tumors as advanced as our patient's, that result in airway compromise and subsequent endotracheal intubation, require surgical resection for definitive management.

\section{Conclusion}

Overall, Hürthle cells have been reported in literature with a diverse array of presentations ranging from benign adenomatous nodules with concomitant Hashimoto thyroiditis to papillary thyroid carcinoma. The standard treatment for Hürthle cell thyroid masses is thyroidectomy. In our case, the patient's mass was untreated for greater than 25 years resulting in respiratory compromise requiring endotracheal tube placement. Intraoperatively, the normal cervical anatomy surrounding the thyroid was completely distorted including displacement of the esophagus, trachea, strap muscles, and left recurrent laryngeal nerve. An extended left cervical incision allowed successful resection of the left superior thyroid lobe, partial resection of the left inferior thyroid lobe and partial resection of the isthmus with visualization of the displaced esophagus and trachea.

\section{References}

1. Agarwal Shipra, Andrey Bychkov, Chan Kwon Jung, et al. (2019) The prevalence and surgical outcomes of hürthle cell lesions in fnas of the thyroid: A multi-institutional study in 6 asian countries. Cancer Cytopathology 127: 181-191.

2. Aytug Serhat (2020) Hurthle cell carcinoma treatment \& management: medical care, surgical care, consultations. Medscape.

3. Cannon Jennifer (2011) The significance of hürthle cells in thyroid disease. The Oncologist 16: 1380-1387.

4. Ali Coskun, Mehmet Yildirim, Nazif Erkan (2014) Substernal goiter: When is a sternotomy required? International Surgery 99: 419-425.

5. Sephton BM (2019) Extracervical approaches to thyroid surgery: evolution and review. Minimally Invasive Surgery 2019: 1-14. 